\title{
LPCAT1 promotes castration resistant prostate cancer progression by increasing PAF release and mRNA synthesis
}

\author{
Bin $X u^{1, *}$, Yajun Cheng ${ }^{1, *}$, Guopeng $\mathbf{Y u}^{1, *}{ }^{1,}$ Wenzhi $\mathbf{L i}^{1}$, Xiang Wan ${ }^{1}$, Juan Zhou ${ }^{1}$ and \\ Zhong Wang ${ }^{1}$ \\ ${ }^{1}$ Department of Urology, Shanghai Ninth People's Hospital, Shanghai Jiaotong University School of Medicine, Shanghai, China \\ *These authors have contributed equally to this work
}

Correspondence to: Zhong Wang, email: zhongwang2000@sina.com

Keywords: LPCAT1; castration resistant prostate cancer (CRPC); Histone H4 palmitorylattion; PAF

Received: November 22, $2017 \quad$ Accepted: January 25, $2018 \quad$ Published: February 09, 2018

Copyright: Xu et al. This is an open-access article distributed under the terms of the Creative Commons Attribution License 3.0 (CC BY 3.0), which permits unrestricted use, distribution, and reproduction in any medium, provided the original author and source are credited.

\section{ABSTRACT}

Our previous study demonstrated that compared with primary prostate cancer (PCa), Lysophosphatidylcholine Acyltransferase1 (LPCAT1) was overexpressed in castration resistant prostate cancer (CRPC) and regulated by androgen through the Wnt-dependent signaling pathway. However, its role in the progression of CRPC remains unclear. The aim of the present study was to explore the roles of LPCAT1 on CRPC progression and the underlying molecular mechanism. It was found that LPCAT1 promoted to the progression of CRPC in terms of proliferation, invasion and migration in vitro and in vivo. The decreased invasion and migration of CRPC cells induced by LPCAT1 silencing could be reversed by platelet activating factor acetylhydrolase (PAF$A H$ ) and PAFR antagonist ABT-491, while the increased invasion and migration of CRPC cells induced by LPCAT1 overexpression can be reversed by exogenous PAF. However, the fluctuated ability of cell proliferation induced by LPCAT1 can't be influenced by PAF-AH or exogenous PAF. Effect of LPCAT1 on increasing growth of CRPC cells by shifting into nucleus to palmitorylate Histone $\mathrm{H} 4$ in an androgen dependent-manner, thus enhancing mRNA synthesis. In addition, the increased expression of LPCAT1 in CRPC cells impaired sensitivity of cancer cells to paclitaxel (PTX) treatment. These results suggest that LPCAT1 may be an effective therapeutic target for CRPC therapy, though more studies are required to deeply elucidate the mechanism underlying role of LPCAT1 in promoting the progression of CRPC.

\section{INTRODUCTION}

Prostatic carcinoma ( $\mathrm{PCa}$ ) is the most common malignancy and the second leading cause of cancer mortality in males [1]. To date, androgen deprivation therapy (ADT) is the gold standard regimen for advanced PCa patients, especially for those with metastasis [2]. Progression of $\mathrm{PCa}$ is androgen dependent but prolonged androgen deprivation generally results in relapse and androgen-independent tumor growth [3]. Commonly, the androgen-independent prostate cancer (AIPC) or castration-resistant prostate cancer (CRPC) is much more malignant and lethal compared to the androgendependent prostate cancer (ADPC) [4-6]. There is limited availablility of clinical treatment strategies for CRPCat present.

Scholars have long puzzled over the mechanism of AIPC development and proposed various hypotheses. In our previous study, we reported the Lysophosphatidylcholine Acyltransferase1 (LPCAT1) as a crucial synthetase of platelet activating factor (PAF) was significantly up-regulated in CRPC tissues and cells, and dihydrotestosterone (DHT) treatment increased the LPCAT1 expression and PAF release in CRPC cells in a 
Wnt/ $\beta$-catenin-dependent manner [7]. However, its role in the progression of CRPC is unknown. In addition, Zhou et al [8] showed that the level of LPCAT1 is higher in PCa and could be used as a predictor of the postprostatectomy outcome. It was also reported that LPCAT1 was overexpressed in breast cancer [9], clear cell renal cell cancer [10] and gastric cancer [11], and contributed to tumor progression. There, LPCAT1 may be a hotspot for exploring new therapeutic targets of cancer therapy.

PAF is an arachidonic acid metabolite and it is generally regarded as a potent pro-inflammatory phospholipid mediator [12]. Increased evidence has shown that it plays an important role in cell proliferation, migration and neoangiogenesis. PAF-acetylhydrolases (PAF-AH) could directly degrade PAF to produce inactive lyso-PAF and acetate by cleaving the sn-2 acetyl residue in the PAF molecule in vivo and in vitro [13]. Our previous work and other published studies [12] showed that PAF played an oncogene-like role, and that PAF signaling was directly involved in CRPC cells invasion and migration [7]. Increased expression of PAF in PCa was mainly attributed to LPCAT1 overexpression via remodeling AA pathway [7]. So we hypothesized that LPCAT1 contributed the progression of CRPC via PAF releasing. In the present study, LPCAT1 Si-RNA was transfected into CRPC C4-2 and PC-3 cells, and the proliferation and mobility of cells were observed in order to explore the potential mechanism. In addition, Exogenous PAF was added into the medium to see whether the effect of LPCAT1 on cell proliferation and mobility could be reversed. Next, LPCAT1 overexpressing plasmid was constructed and transfected into PC-3 cells. PAFR antagonist ABT491 and human recombinant Platelet Activating Factor Acetylhydrolase (PAH-AH) was used to verify the effect of LPCAT1 overexpression. Finally, effect of LPCAT1 on CRPC cells chemotherapy was observed.

\section{RESULTS}

\section{Effect of LPCAT1 knockdown on the progression of CRPC}

Although we previously demonstrated that LPCAT1 was overexpressed in CRPC and regulated by androgens, its role in CRPC has not been studied. To explore its potential role, CRPC C4-2 and PC-3 cells were transfected with LPCAT1 Si-RNA, and the transfection efficiency is shown in Figure 1A. Next, we found that LPCAT1 silencing significantly decreased the proliferation of either C4-2 cells or PC-3 cells at 48 and 72 h (Figure 1B). Furthermore, LPCAT1 silencing significantly suppressed the cells migration and invasion (Figure $1 \mathrm{C}$ and $1 \mathrm{D}$ ). To determine whether $t$ LPCAT1 silencing-induced effect was regulated by down-regulating the PAF expression, the PAF concentration was measured in LPCAT1 silencing group and the control group. It was found that the PAF concentration was decreased after LPCAT1 silencing (Figure 1E). Therefore, cells in Si-LPCAT1 group were exposed to exogenous PAF, and consistent with our previous results, we found that exogenous PAF with increasing concentration $\left(10^{-8}-10^{-6} \mathrm{M}\right)$ has no effect on proliferation of cells with si-LPCAT1(Figure 1F). On the contrary, cell mobility suppressed by LPCAT1 silencing could be reversed by exogenous PAF in a concentrationdependent manner, which can be blocked by PAF receptor antagonist ABT-491 (Figure $1 \mathrm{G}$ and $1 \mathrm{H}$ ). Above results suggest that LPCAT1 silencing inhibited CRPC cell migration and invasion by PAF-PAFR signaling pathway. Whether the growth of CRPC cells suppressed by LPCAT1 was regulated by another potential molecular mechanism needs us to be illuminated.

\section{Effect of LPCAT1 overexpression on CRPC cells mobility}

To further explore the role of LPCAT1 in CRPC progression, LPCAT1 was transfected into PC-3 cells and the transfection efficiency was measured (Figure 2A). It was found that the ability of cell migration and invasion was increased in LPCAT1 overexpression group as compared with that in the control group (Figure 2B). Furthermore, the PAF concentration was determined in both groups. It was found that the PAF concentration was increased in LPCAT1 overexpression group (Figure 2C). To explore whether the effect was regulated by increasing PAF releasing, different concentrations of the PAF deactivator, PAF-AH and PAF receptor antagonist, ABT491 were added into cell cultures to degrade PAF or inhibit PAFR, respectively. The degradation efficiency of PAF-AH was measured in Figure 3E. It was found that the increased ability of PC-3 cell migration and invasion induced by LPCAT1 overexpression could be reversed by PAF-AH (Figure 2D), and ABT-491(Figure 2E), with concentration dependence. These results suggest that LPCAT1 increased CRPC cells migration and invasion via promoting PAF-PAFR signaling pathway.

\section{Effect of LPCAT1 overexpression on CRPC cell growth}

Firstly, PC-3 cells with LPCAT1 overexpression were constructed and the transfection efficiency was measured (Figure 2A). It was found that cell proliferation was increased in LPCAT1 overexpressed group as compared with in the control group (Figure 3A). Consistent with above results, colony formation assay also showed that cells growth were promoted by LPCAT1 (Figure 3B). Furthermore, cell cycle assay showed that the number of cells in $\mathrm{G} 1$ and $\mathrm{S}$ phase was decreased and the number of cells in G2 phase was increased in LPCAT1 overexpressed group as compared with the control group (Figure 3C). Finally, a xenograft 
tumor model was conducted to evaluate the growth of CRPC cells induced by LPCAT1. It was found that the mean tumor weight was significantly increased in LPCAT1 overexpression tumors compared with control tumors $(\mathrm{p}<0.05)$ (Figure 3D). Figure $2 \mathrm{C}$ showed that PAF concentration significantly increased in LPCAT1 overexpression group compared with the control group. To explore whether the effect was regulated by increasing PAF releasing, different concentration of PAF-AH were added into cell cultures to degrade PAF. The results in Figure 3E showed that the increased PAF concentration in LPCAT1 group was reversed by PAF-AH compared with the control group. Subsequent cell proliferation assay showed that the increased cell proliferation in LPCAT1 overexpression group was not affected by exogenous PAF-AH. These results suggest that the effect of increased proliferation induced by LPCAT1 is not regulated by PFA releasing.
A

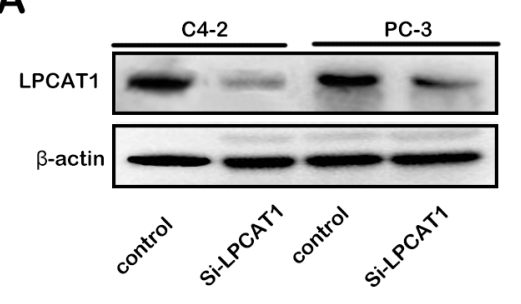

C

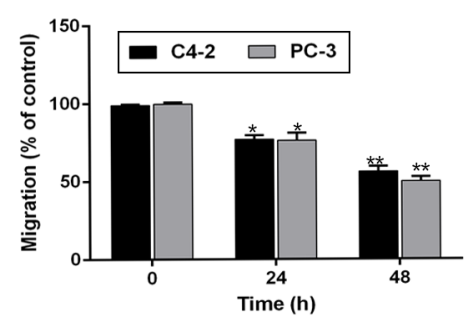

E

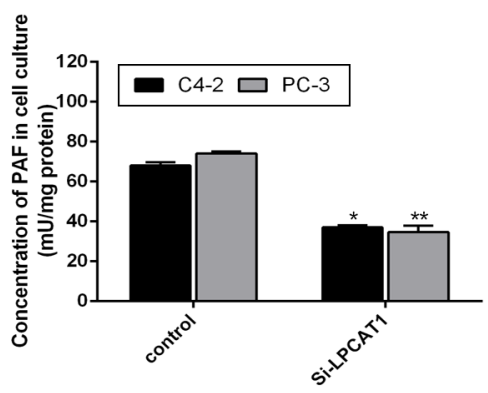

G

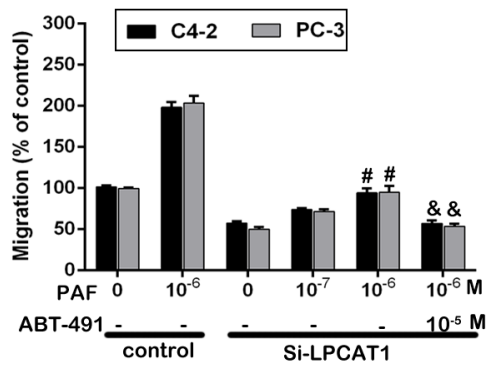

B

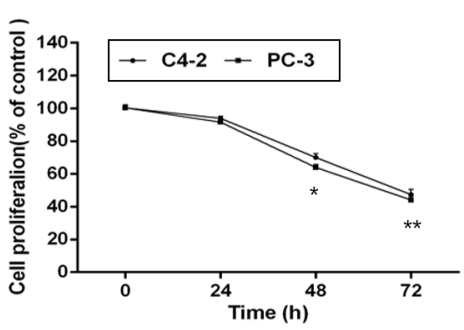

D

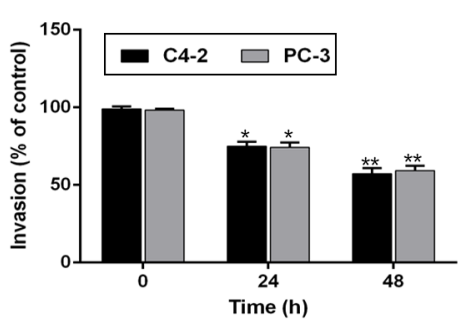

$\mathbf{F}$

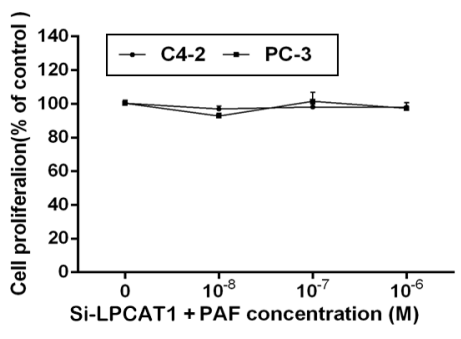

H

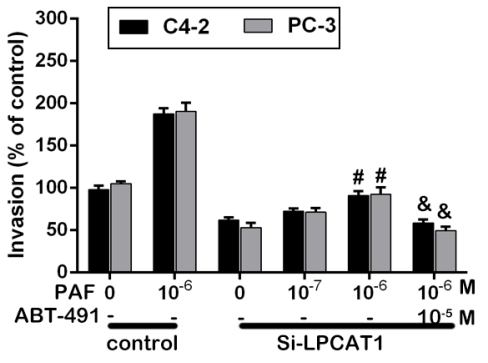

Figure 1: The effect of LPCAT1 knockdown on CRPC progression. (A) LPCAT1 Si-RNA was transfected into C4-2 and PC-3 cells, and transfection efficiency was demonstrated. (B) The ability of cell proliferation was measured at 0, 24, 48 and $72 \mathrm{~h}$ after transfection. (C) and (D) The ability of cell migration and invasion were determined at 0, 24 and $48 \mathrm{~h}$ after transfection. (E) The PAF concentration in cell cultures of Si-LPCAT1 group and control at $48 \mathrm{~h}$ after transfection. (F) Cell proliferation assay were measured at $48 \mathrm{~h}$ after C4-2 and PC-3 cells in Si-LPCAT1 group were treated with increasing concentration of PAF $\left(10^{-8}-10^{-6} \mathrm{M}\right)$ and the control group. (G) and (F) The effect of the increased concentration of PAF $\left(10^{-8}-10^{-6} \mathrm{M}\right)$ on migration and invasion inSi-LPCAT1 group and control group, and ABT-491 $\left(10^{-5}\right.$ $\mathrm{M})$ was used to pretreat cells induced by PAF $\left(10^{-6} \mathrm{M}\right)$. Values are presented as mean percent control \pm s.e.m or mean \pm s.e.m. ${ }^{*} \mathrm{P}<0.05$ and ${ }^{* *} \mathrm{P}<0.01$ compared with control; ${ }^{\#} \mathrm{P}<0.05$ and ${ }^{\# \#} \mathrm{P}<0.01$ compared with Si-LPCAT1 treated with PAF $(0 \mathrm{M}) ;{ }^{\text {\& }} \mathrm{P}<0.05$ and \&\& $\mathrm{P}<0.01$ compared with Si-LPCAT1 treated with PAF $\left(10^{-6} \mathrm{M}\right)$. 


\section{LPCAT1 shifts into the nucleus in the presence of androgen and enhances mRNA synthesis}

Interestingly, although CRPC cells mobility regulated by LPCAT1 could be reversed by PAF-PAFR signaling intervention, the increased proliferation of CRPC cells induced by LPCTA1 was not affected by PAF releasing. Previous studies demonstrated that LPCAT1 increased the mRNA synthesis by shifting into the nucleus [15]. It was found that LPCAT1 expression in nucleus was increased in PC-3 cells transfected with LPCAT1 compared the control cells cultured in 1640 medium containing a certain amount of androgen (Figure $4 \mathrm{~A}$ ). We postulated that androgen may participate in the process of LPCAT1 nucleus shift. Therefore, cells were treated with increasing concentration of DHT. It was found thatLPCAT1 expression in cell nucleus increased with androgen concentration increasing (Figure 4B). Next, LPCAT1 overexpressing cells and control were simultaneously cultured with DHT $\left(10^{-6} \mathrm{M}\right)$, DHT $\left(10^{-6} \mathrm{M}\right)$ + Flu or DHT (0 M). It was found that cell proliferation was increased in LPCAT1 overexpressing cells compared with control cells when cultured with DHT $\left(10^{-6} \mathrm{M}\right)$, and Flu could reverse this effect induced by DHT $\left(10^{-6}\right.$ M) (Figure 4C). Moreover, tumorigenesis assay showed that there was no significant difference in tumor weight between LPCAT1 overexpressing group and control when male nude mice was castrated (Figure 4D). These results suggest that the effect of LPCAT1 on the increasing growth of CRPC cells was through shifting into the nucleus in an androgen-dependent manner. To further probe the potential mechanism of this process, activated RNA polymerase (RNAP) II and RNA synthesis was measured in LPCAT1 overexpressing cells and control cells after androgen stimulation. We showed that RNAP II activation and RNA synthesis was increased in LPCAT1 overexpressing group compared with the control group (Figure 4E). Previous studies reported that above process was regulated by histone H4 O-palmitorylation [14]. Therefore, histone H4 S47A mutation was designed and transfected. It was found that the increased RNAP II activation and RNA synthesis could be reversed by $\mathrm{H} 4$ mutation (Figure 4F), suggesting that histone $\mathrm{H} 4 \mathrm{O}$-palmitorylation was necessary for the process of increasing RNA synthesis via LPCAT1 shifting into cell nucleus in CRPC cells.

\section{The effect of paclitaxel is regulated by LPCAT1 expression}

Although LPCAT1 enhanced the chemotherapy sensitivity of lung cancer cell to cisplatin, the effect of LPCAT1 on CRPC cells chemotherapy is unknown. LPCAT1-overexpressing and control cells were treated with increasing concentration paclitaxel. Cell viability assay showed that LPCAT1 overexpression decreased the sensitivity of CRPC cells to paclitaxel (Figure 5A). In addition, it induced the less cell apoptosis compared with the control cells in presence of paclitaxel $(\mathrm{P}<0.05)$ (Figure 5B).Next, tumorigenesis assays was performed to determine whether LPCAT1 enhanced the resistant
A

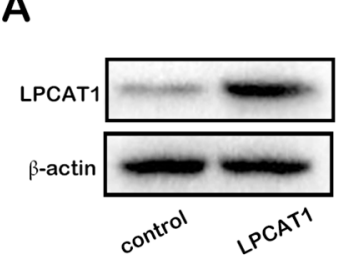

D

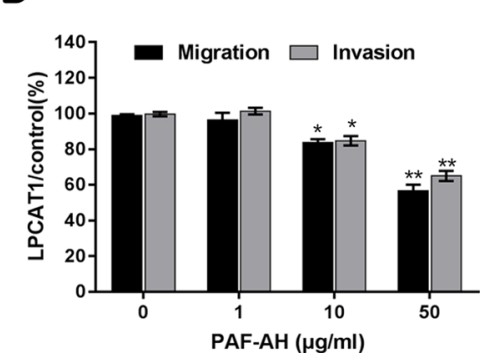

B

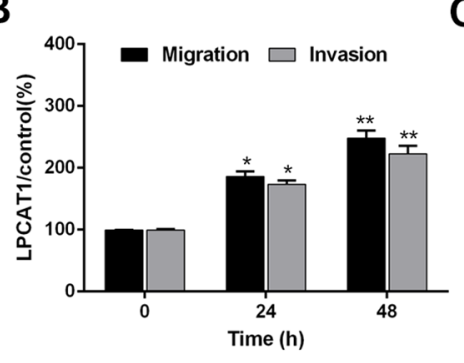

$\mathrm{E}$
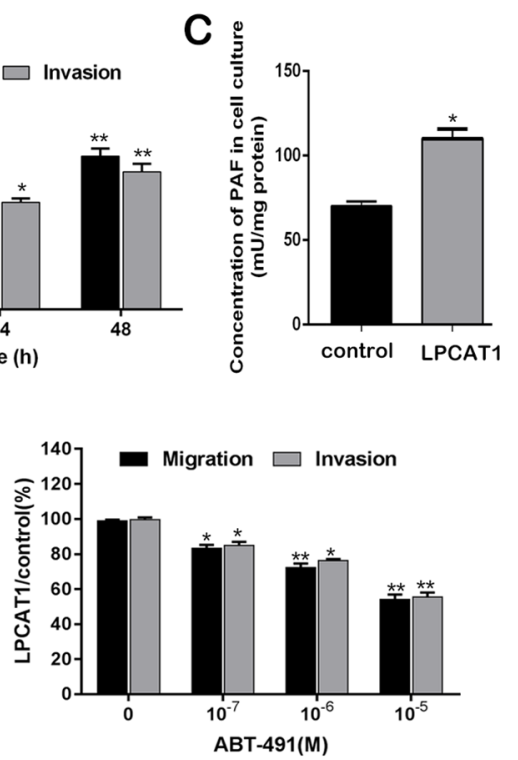

Figure 2: The effect of LPCAT1 overexpression on CRPC cell mobility. (A) LPCAT1 was transfected into PC-3 cells, and the transfection efficiency was demonstrated. (B) the ability of cell migration and invasion was determined at 0, 24 and $48 \mathrm{~h}$. (C) The PAF concentration in cell cultures of LPCAT1 and control groups. (D) and (E) The effect of increased concentration of PAF-AH (1-50 $\mu \mathrm{g} / \mathrm{ml})$ and ABT-491 on migration and invasion inLPCAT1 and control groups. Values are presented as mean percent control \pm s.e.m or mean \pm s.e.m. ${ }^{*} \mathrm{P}<0.05$ and ${ }^{* *} \mathrm{P}<0.01$ compared with control. 
effect of paclitaxel on CRPC cells. Cells stably expressing exogenous LPCAT1 or control vector were injected subcutaneously into nude mice, with or without paclitaxel treatment. During the 3-week period of tumor growth, before paclitaxel administration, LPCAT1 expression appeared to increase tumor volumes but insignificantly (Figure 5C), while tumor growth was significantly suppressed after administration of paclitaxel treatment, this suppressive effect was much more profound in tumors of the control group than in (Figure 5D). In addition, tumors were also weighed (Figure $3 \mathrm{~B}$ ), and the results showed that LPCAT1 decreased the sensitivity of paclitaxel to CRPC cells. These results indicate that the increased expression of LPCAT1 impaired sensitivity of CRPC cells to paclitaxel treatment in the xenograft model.

\section{DISCUSSION}

In the present study, we explored the role of LPCAT1 in CRPC progression and potential molecular mechanism, finding that LPCAT1 promoted proliferation, invasion and migration of CRPC cells in vitro and in vivo, and the increased or decreased invasion and migration of CRPC cells induced by LPCAT1 overexpression or silencing can be reversed by PAF-PAFR pathway inhibition, or exogenous PAF, respectively. However, the fluctuated ability of cell proliferation induced by LPCAT1 can't be influenced by PAF-AH or exogenous PAF. LPCAT1 increased growth of CRPC cells by shifting into the nucleus to palmitorylate Histone $\mathrm{H} 4$ in an androgendependent manner, thus enhancing mRNA synthesis. Meanwhile, the increased expression of LPCAT1 in CRPC cells reduced sensitivity of cancer cells to paclitaxel treatment.

Although PCa is a kind of indolent cancer, and localized forms can be well managed and successfully treated, progression of primary $\mathrm{PCa}$ into CRPC remains a culprit killing the thousands of men every day [15]. PCa remains the most frequently diagnosed cancer and the second leading cause of cancer-death in men in western

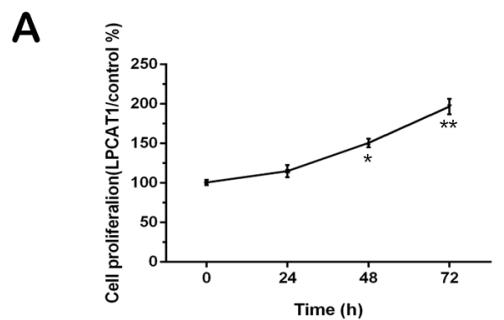

C
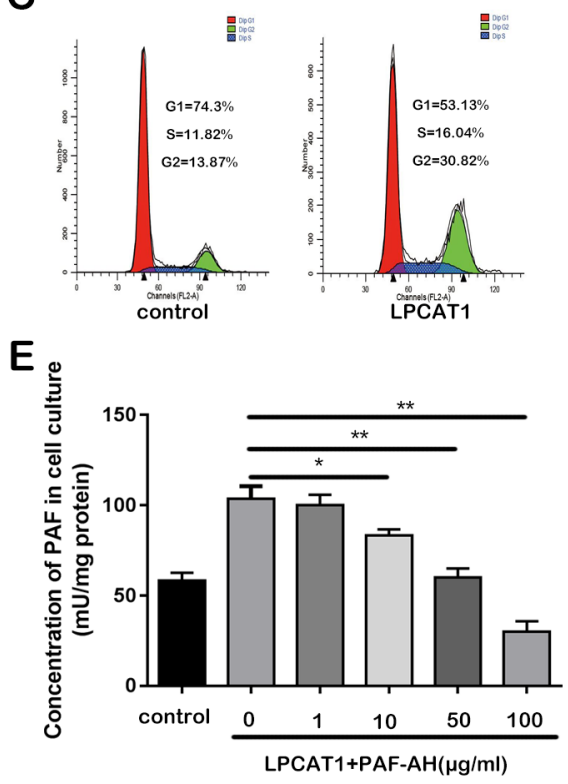

B

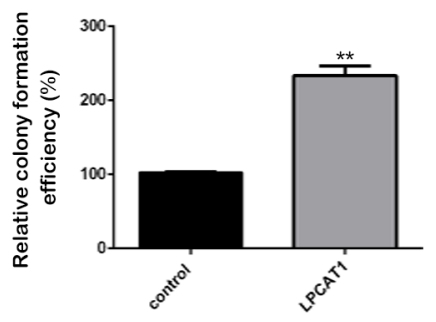

D

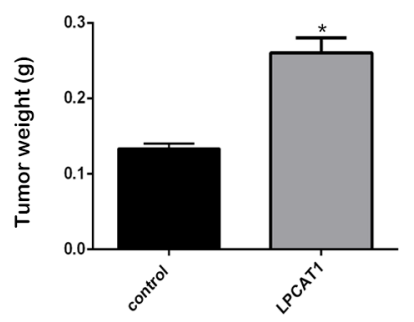

$\mathbf{F}$

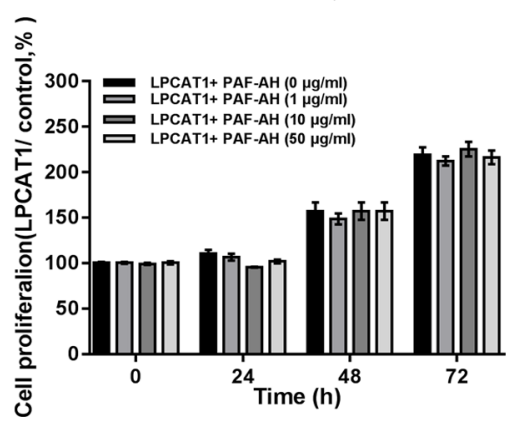

Figure 3: The effect of LPCAT1 overexpression o CRPC cell growth. (A) The ability of Cell proliferation was measured at 0, 24, 48 and $72 \mathrm{~h}$. (B) Colony formation efficiency was measured in LPCAT1 and control groups. (C) cell cycle was measured in LPCAT1 and control groups. (D) Tumorigenicity assay in nude mice was performed and tumor weight in LPCAT1 and control groups was measured. (E) The PAF concentration was measured after PAF-AH treatment $(\mathbf{F})$ the ability of Cell proliferation in LCPAT1 with different concentration PAF-AH treatment and control was measured at $0,24,48$ and $72 \mathrm{~h}$. Values are presented as mean percent control \pm s.e.m or mean \pm s.e.m. ${ }^{*}$ $\mathrm{P}<0.05$ and $^{* *} \mathrm{P}<0.01$ compared with control. 
countries [16]. Therefore, it needs to identify novel therapeutic targets to improve prognosis and treatment of this cancer. LPCAT1 overexpression in CRPC tissues is known to contribute to the proliferation, invasion and migration of CRPC cells in vitro and in vivo, as well as the progression of CRPC. PAF is a phosphatidylcholinederived molecule that plays an important role in inflammatory processes and various types of cancer progression by binding PAFR, and ultimately leading to activation of signal pathway [17]. PAF can be biosynthesized by a remodeling pathway: LPCAT1 converted lyso-PAF into PAF. In addition, some recent studies showed that PAF could also be biosynthesized by another lyso-PAF acetyltransferase enzymes, LPACT2 by lyso-PAF and acetyl-CoA as substrates [18]. A systems genetics approach analysis revealed that LPCAT2 expression was positively correlated with aggressive $\mathrm{PCa}$ [19]. However, its role in PCa and PAF production in $\mathrm{PCa}$ is unclear and needs us to further exploration.
Although PAF was reported to promote the migration and proliferation of breast cancer, esophageal squamous cell carcinoma and ovarian cancer mainly via activating PAFR signaling pathway [20], our previous studies [7] showed that PAF was only involved PC-3 cells invasion and migration. The increased or decreased invasion and migration of CRPC cells induced by LPCAT1 overexpression or silencing can be reversed by $\mathrm{PAF}$ pathway inhibition or exogenous PAF, respectively. These results suggest that LPCAT1 promoted mobility of PC-3 cells by increasing PAF.

To determine the mechanism underlying the role of LPCAT1 in increasing proliferation of CRPC cells, LPCAT1 expression in the nucleus was measured. It was found that LPCAT1 shifts into nucleus in LPCAT1 overexpression group as compared with the control group. LPCAT1 was mainly localized in the cell cytoplasm and initially translocated into the nucleus after various stimulation, including bacterial pathgens, LPS and $\mathrm{Ca}^{2+}$
A

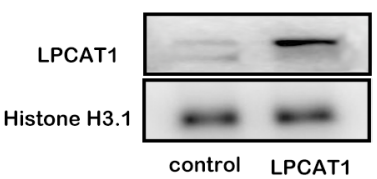

C ำ

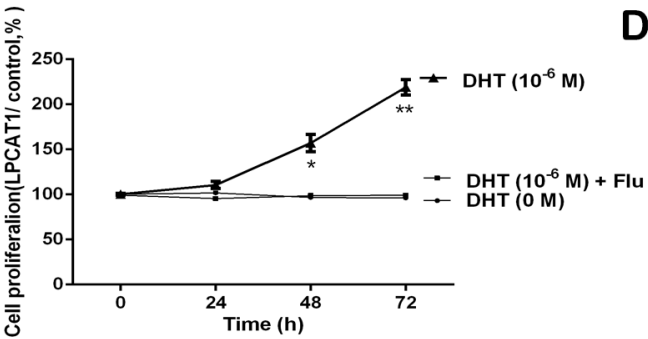

B

D
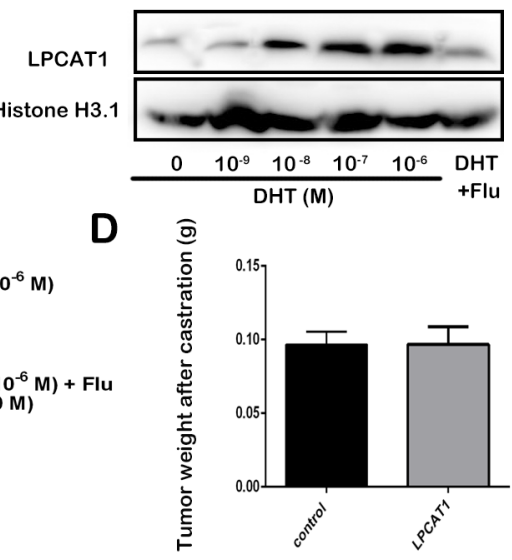

$F$

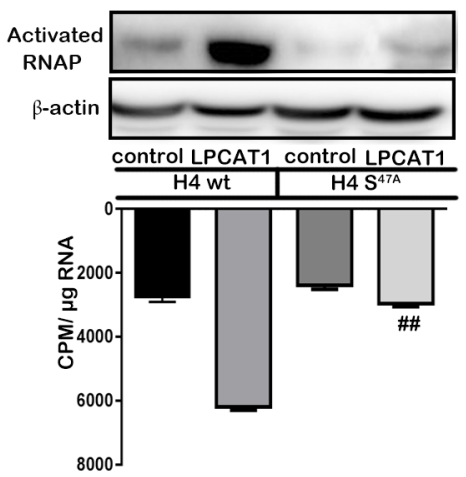

Figure 4: LPCAT1 shifts into the nucleus in the presence of androgen and enhancs mRNA synthesis. (A) Intra-nuclear LPCAT1 expression in LPCAT1 and control groups and cells were cultured with ordinary FBS. (B) The effect of the increased concentrations of DHT on intra-nuclear LPCAT1 expression of PC-3 cells cultured in Dextran Stripped FBS was detected. (C) The effect of DHT (10 6 M), DHT $\left(10^{-6} \mathrm{M}\right)+$ Flu and DHT $(0 \mathrm{M})$ on the ability of PC-3 cells cultured in Dextran Stripped FBS was detected. (D) Tumor weight in nude mice with LPCAT1 and control after castration. (E) Activated RNA polymerase (RNAP) II expression (up) and total RNA synthesis (down) were measured in LPCAT1 and control group cells with ordinary FBS culture. (F) Histone H4 S47A mutation was designed and transfected into LPCAT1 and control groups, and activated RNA polymerase (RNAP) II expression (up) and total RNA synthesis (down) were measured in WT and mutation group. Values are presented as mean percent control \pm s.e.m or mean \pm s.e.m. ${ }^{*} \mathrm{P}<0.05$ and ${ }^{* *} \mathrm{P}<0.01$ compared with control. ${ }^{\#}<0.05$ and ${ }^{\#} \mathrm{P}<0.01$ compared with LPCAT1 overexpressing cells in WT group. 
$[14,21]$. Then, nuclear Lpcat1 subsequently catalyzes histone palmitoylation through direct binding to histone protein, thus increasing the total RNA synthesis. With LPS stimulation, histone palmitoylation could be a novel epigenetic mark that controls pro-inflammatory gene transcription [21]. Therefore, we hypothesized that LPCAT1 may catalyze histone palmitoylation by shifting into nucleus, and ultimately inceasing RNA synthesis increasing under androgen stimulation. LPCAT1 expression in the nucleus was increased under androgen stimulation in a concentration-dependent manner. Meanwhile, the increased proliferation induced by LPCAT1 could be reversed by androgen depletion in vivo and in vitro, suggesting that the increased proliferation induced by LPCAT1 was through shifting into nucleus in an androgen-dependent manner. It is possible that the increase in $\mathrm{Ca}^{2+}$ influx promoted by DHT [22] is a factor for LPCAT1 re-translocation for the activation of RNA synthesis.

Palmitoylation (S-, N- and O-palmitoylation), as an important kind of post-translational modification, exists ubiquitously in eukocytes. Compared with S palmitoylation, O-palmitoylation is less studied. A recent study reported that O-palmitoylation occurred in Wnt family proteins to regulate Wnt protein secretion, ultimately leading to activation or inactivation of the Wnt pathway [23]. We showed that histone H4 O-palmitoylation induced by LPCAT1 increased the total RNA synthesis. Histone family protein, including H2A, $\mathrm{H} 2 \mathrm{~B}, \mathrm{H} 3$, and $\mathrm{H} 4$, play important roles in various diseases, including cancer, by post-translational modification [24]. The progression of $\mathrm{PCa}$ or CRPC could be promoted by epigenetic modifications of Histone with acetylation, phosphorylation, or methylation [25]. Multiple histone deacetylase inhibitors have been shown to effectively suppress the progression of CRPC alone or combination with other chemotherapy drugs, some of which have been studies in clinical trials [26, 27]. Histone H4 O-palmitoylation may prove to be an effective therapeutic target for CRPC therapy, although it needs more studied to illustrate tis potential function on the progression of CRPC.
A

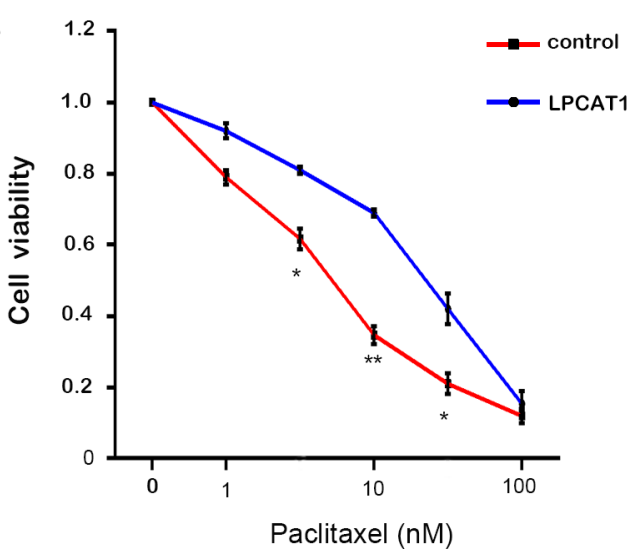

B
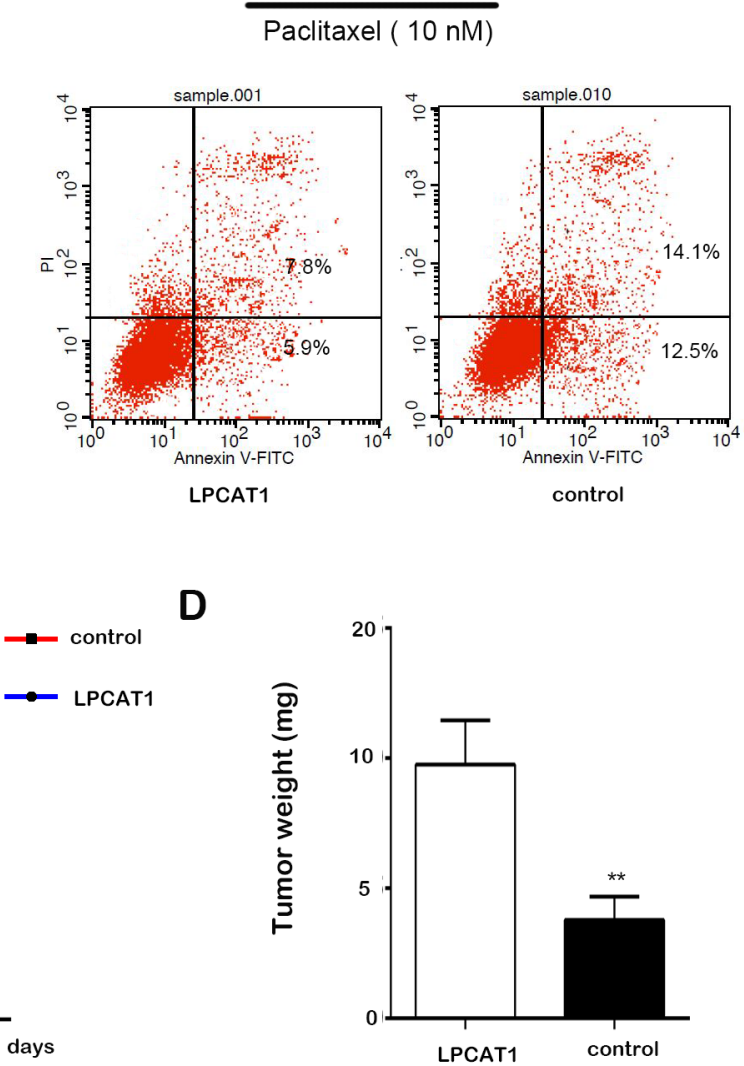

Figure 5: The effect of paclitaxel is regulated by LPCAT1 expression. (A) Cell viability was determined in LPCAT1 and control groups with the increasing concentration of paclitaxel treatment (1, 10 and $100 \mathrm{nM})$. (B) Cell apoptosis in LPCAT1 and control group with paclitaxel $(10 \mathrm{nM})$ treatment. (C) and (D) cells were injected into nude mice to initiate tumorigenesis, with paclitaxel treatment administered 14 days post inoculation. Tumor volumes at different time points (C) and tumor weights (D) at excision. Values are presented as mean \pm s.e.m. ${ }^{*} \mathrm{P}<0.05$ and ${ }^{* *} \mathrm{P}<0.01$ compared with control. 


\section{MATERIALS AND METHODS}

\section{Ethics statement}

This experiment was approved by the institutional Ethical Committee of Shanghai Ninth Peoples' Hospital (Shanghai, China). All the animals received humane care in accordance with the recommendations of the Guide for the Care and Use of Laboratory.

\section{Establishment of the experimental model}

For tumorigenesis assay in nude mice, PC-3 cells were transfected with LPCAT1 plasmid or control, and re-suspended in Matrigel solution (BD, USA) and an equal volume PBS. The suspension was then injected subcutaneously into the flanks of 8 -week-old male $\mathrm{BALB} / \mathrm{c}$ nude mice at $3 \times 10^{6}$ cells per site. All mice were sacrificed at 6 weeks, and the tumors were removed and weighed.

To evaluate the effect of androgen on the tumor formation ability of cells transfected with LPCAT1 or control, the testis was removed at 2 weeks after subcutaneous injection of the cells. All mice were sacrificed at 6 weeks, and the tumosr were removed and weighed.

To evaluate the sensitivity of cells overexpressing LPCAT1 or control to chemotherapy, mice were administered with daily intra-peritoneal (i.p.) injections of paclitaxel $(20 \mathrm{mg} / \mathrm{kg} \mathrm{b.w})$ or the solvent DMSO 14 days after cell inoculation for 5 consecutive day as previously described [28]. At the end of the tumorigenesis assay, mice were sacrificed previously. Tumor volumes were measured every second day and lastly, mice were sacrificed and the tumors were removed and weighed.

\section{Cell culture}

C4-2 and PC-3 cells were kindly purchased from Stem Cell Bank, Chinese Academy of Sciences (Shanghai, China), and cultured in the RPMI-1640 medium supplemented with $10 \%$ fetal bovine serum (FBS) (Gemini, 100-700) or Dextran Stripped FBS (Gemini, 100-119) and 1\% penicillin/streptomycin (P/S). All cells were maintained in an incubator at $37^{\circ} \mathrm{C}$ with $5 \% \mathrm{CO}^{2}$.

\section{Cell transfection}

For cell interference, LPCAT1 Si-RNA (SR312680) was purchased from Origene biotech (Beijing, China). In Brief, $2 \times 10^{5}$ cells were maintained in a 6 -well plate. After $12 \mathrm{~h}$, siRNA was transfected into cells using Roche reagent (04476093001) according to the manufacturer's protocol. At $48 \mathrm{~h}$ after transfection, cells were harvested to measure the efficacy of transfection. For plasmid transfection, full-length human LPCAT1 cDNA (a gift from Han's Lab) was cloned into a pCDH-CMV-MCS-
EF1-puro vector. The LPCAT1 expression or a control plasmid was cotransfected with pMD.2G and PSPAX2 into HEK-293FT cells with Lipofectamine LTX (Invitrogen) according to the manufacturer's protocol. Viruses in the culture supernatants were harvested $48 \mathrm{~h}$ after transfection, and PC-3 cells were infected twice at 24-h intervals. After infection, cells were selected with puromycin.

\section{Mutagenesis}

Histone H4 S47A mutation was designed and structured in GenePharma Co., Ltd (Suzhou, China) as previously described.

\section{Cell proliferation, migration and invasion assay}

Cell proliferation was evaluated by MTS cell proliferation assay, and cell mobility was evaluated by transwell assay as previously described [7].

\section{Cell cycle and cell apoptosis}

For cell cycle assay, cells were harvested, fixed in $70 \%$ ethanol at $4^{\circ} \mathrm{C}$ for at least $24 \mathrm{~h}$, re-suspended in PBS, incubated with propidium iodide (PI) (Abcam, USA) in the presence of RNase A for 30 min in the dark, and finally subjected to flow cytometry analysis. For cell apoptosis assay, After incubation with paclitaxel for 48 hours, cells were collected, washed with cold PBS, re-suspended in $100 \mu 1$ of $1 \times$ Annexin V binding buffer, and stained with Annexin V and PI (BD Pharmingen) by adding $5 \mu \mathrm{l}$ to each tube and incubating for $15 \mathrm{~min}$ at room temperature in the dark for flow cytometry analysis to detect cell apoptosis

\section{Western blotting}

For whole protein extraction, cells were harvested, centrifuged at 1,000 rpm for $10 \mathrm{~min}$, put into lysis buffer for $30 \mathrm{~min}$ at $4^{\circ} \mathrm{C}$, and centrifuged at $13,000 \mathrm{rpm}$ at $4^{\circ} \mathrm{C}$ for $15 \mathrm{~min}$; for nuclear protein extraction, Nuclear and Cytoplasmic Protein Extraction Kit (Beyotime, China, P0027) was used to extract nuclear protein according to the instruction. The supernatant was collected, and 1 protein was measured by using a Bradford kit (Beyotime, China). Next, protein was separated by SDS-PAGE and then transferred onto PVDF Transfer Membranes (Millipore, Billerica, MA, USA). The membranes were then blocked using 5\% non-fat milk in TBST for $1 \mathrm{~h}$ at room temperature and then were incubated with primary antibodies (LPCAT1, Abcam, ab214034; Histone H3.1, Cell Signaling Technology, 9717; active RNA polymerase II, Millipore, 04-1570; $\beta$-actin, Abcam, ab8227) at $4^{\circ} \mathrm{C}$ overnight. After three washes with TBST, the membranes were incubated with secondary antibodies conjugated with HRP for $1 \mathrm{~h}$ at room temperature. Finally, the blots were visualized by a Super Signal West Pico Chemiluminescent 
Substrate (Thermo Fisher Scientific, Massachusetts, MA, USA) according to the manufacturer\&s protocol.

\section{Pulse-chase labeling of RNA and RNA Isolation}

Cells in exponential growth were pulse-chaselabeled with $1 \mu \mathrm{Ci}$ of $\left[{ }^{32} \mathrm{P}\right]$ UTP for $2 \mathrm{~h}$. Total RNA was extracted from the cells using TRIzol. The radioactivity of the isolated RNA was measured by scintillation counting.

\section{ELISA measurement of PAF}

PAF concentration in cell medium was measured by a ELISA Kit (Westang Biotech, China) according to the manufacture's protocol. For human recombinant Platelet Activating Factor Acetylhydrolase (PAH-AH) (PeproTech EC Ltd., USA) treatment, cell culture was collected at $24 \mathrm{~h}$ after PAH-AH addition.

\section{Statistical analysis}

For illustrative purposes, the results are presented as the mean percent control \pm s.e.m. One-way ANOVA followed by the Student-Newman-Keuls test was used to assess significant differences between each two groups. Significance was set at $\mathrm{P}<0.05$.

\section{CONCLUSION}

LPCAT1 was overexpressed in CRPC and contributed to its progression. LPCAT1 overexpression increased the mobility of CRPC cells by enhancing PAF releasing. Meanwhile, increased proliferation induced by LPCAT1 mainly attributed to itself via shifting into nucleus to palmitorylate Histone $\mathrm{H} 4$ in an androgen dependent-manner, thus enhancing mRNA synthesis.

\section{CONFLICTS OF INTEREST}

The authors have no conflicts of interest to disclose.

\section{FUNDING}

This work is supported by the National Natural Science Foundation of China (No.81472398) and Shanghai Rising-Star Program (No.15QA1404900).

\section{REFERENCES}

1. Gronberg H. Prostate cancer epidemiology. Lancet. 2003; 361: 859-64.

2. Wang HQ, Yang B, Xu CL, Wang LH, Zhang YX, Xu B, Ji JT, Sun YH. Differential phosphoprotein levels and pathway analysis identify the transition mechanism of $\mathrm{LNCaP}$ cells into androgen-independent cells. Prostate. 2010; 70:508-17.
3. Moul JW, Evans CP, Gomella LG, Roach M 3rd, Dreicer R. Traditional approaches to androgen deprivation therapy. Urology. 2011; 78:S485-93.

4. Saraon P, Drabovich AP, Jarvi KA, Diamandis EP. Mechanisms of Androgen-Independent Prostate Cancer. EJIFCC. 2014; 28;25:42-54.

5. Fiñones RR, Yeargin J, Lee M, Kaur AP, Cheng C, Sun $\mathrm{P}$, Wu C, Nguyen C, Wang-Rodriguez J, Meyer AN, Baird SM, Donoghue DJ, Haas M. Early human prostate adenocarcinomas harbor androgen-independent cancer cells. PLoS One. 2013; 8:e74438.

6. Komiya A, Shimbo M, Suzuki H, Imamoto T, Kato T, Fukasawa S, Kamiya N, Naya Y, Mori I, Ichikawa T. Oral low-dose dexamethasone for androgen-independent prostate cancer patients. Oncol Lett. 2010; 1:73-79.

7. Xu B, Gao L, Wang L, Tang G, He M, Yu Y, Ni X, Sun Y. Effects of platelet-activating factor and its differential regulation by androgens and steroid hormones in prostate cancers. Br J Cancer. 2013; 109:1279-86.

8. Zhou X, Lawrence TJ, He Z, Pound CR, Mao J, Bigler SA. The expression level of lysophosphatidylcholine acyltransferase 1 (LPCAT1) correlates to the progression of prostate cancer. Exp Mol Pathol. 2012; 92:105-10.

9. Abdelzaher E, Mostafa MF. Lysophosphatidylcholine acyltransferase 1 (LPCAT1) upregulation in breast carcinoma contributes to tumor progression and predicts early tumor recurrence. Tumour Biol. 2015; 36:5473-83.

10. Du Y, Wang Q, Zhang X, Wang X, Qin C, Sheng Z, Yin H, Jiang C, Li J, Xu T. Lysophosphatidylcholine acyltransferase 1 upregulation and concomitant phospholipid alterations in clear cell renal cell carcinoma. J Exp Clin Cancer Res. 2017; 36:66.

11. Uehara T, Kikuchi H, Miyazaki S, Iino I, Setoguchi T, Hiramatsu Y, Ohta M, Kamiya K, Morita Y, Tanaka H, Baba S, Hayasaka T, Setou M, Konno H. Overexpression of Lysophosphatidylcholine Acyltransferase 1 and Concomitant Lipid Alterations in Gastric Cancer. Ann Surg Oncol. 2016; 23:S206-13.

12. Tarui M, Shindou H, Kumagai K, Morimoto R, Harayama T, Hashidate T, Kojima H, Okabe T, Nagano T, Nagase T, Shimizu T. Selective inhibitors of a PAF biosynthetic enzyme lysophosphatidylcholine acyltransferase 2. J Lipid Res. 2014; 55:1386-96.

13. Wu Y, Wang L, Dai C, Ma G, Zhang Y, Zhang X, $\mathrm{Wu} \mathrm{Z}$. Neuroprotection by platelet-activating factor acetylhydrolase in a mouse model of transient cerebral ischemia. Neurosci Lett. 2014; 558:26-30.

14. Zou C, Ellis BM, Smith RM, Chen BB, Zhao Y, Mallampalli RK. Acyl-CoA:lysophosphatidylcholine acyltransferase I (Lpcat1) catalyzes histone protein O-palmitoylation to regulate mRNA synthesis. J Biol Chem. 2011; 286:28019-25.16.

15. Zhu X, Albertsen PC, Andriole GL, Roobol MJ, Schröder FH, Vickers AJ. Riskbased prostate cancer screening. Eur Urol. 2012; 61:652-61. 
16. Ferlay J, Shin HR, Bray F, Forman D, Mathers C, Parkin DM. Estimates of worldwide burden of cancer in 2008: GLOBOCAN 2008. Int J Cancer. 2010; 127:2893-917.

17. Jan CR, Chao YY. Novel effect of Y-24180, a presumed specific platelet activation factor receptor antagonist, on $\mathrm{Ca} 2+$ levels and growth of human prostate cancer cells. Cell Signal. 2004; 16:959-65.

18. Shindou H, Shiraishi S, Tokuoka SM, Takahashi Y, Harayama T, Abe T, Bando K, Miyano K, Kita Y, Uezono Y, Shimizu T. Relief from neuropathic pain by blocking of the platelet-activating factor-pain loop. FASEB J. 2017; 31:2973-2980.

19. Williams KA, Lee M, Hu Y, Andreas J, Patel SJ, Zhang S, Chines P, Elkahloun A, Chandrasekharappa S, Gutkind JS, Molinolo AA, Crawford NP. A systems genetics approach identifies CXCL14, ITGAX, and LPCAT2 as novel aggressive prostate cancer susceptibility genes. PLoS Genet. 2014; 10:e1004809.

20. Ji W, Chen J, Mi Y, Wang G, Xu X, Wang W. Plateletactivating factor receptor activation promotes prostate cancer cell growth, invasion and metastasis via ERK1/2 pathway. Int J Oncol. 2016; 49:181-8.

21. Ellis B, Kaercher L, Snavely C, Zhao Y, Zou C. Lipopolysaccharide triggers nuclear import of Lpcat1 to regulate inducible gene expression in lung epithelia. World J Biol Chem. 2012; 3:159-66.

22. Al-Bakheit A, Traka M, Saha S, Mithen R, Melchini A. Accumulation of Palmitoylcarnitine and Its Effect on ProInflammatory Pathways and Calcium Influx in Prostate Cancer. Prostate. 2016; 76:1326-37.
23. Gao X, Hannoush RN. Single-cell imaging of Wnt palmitoylation by the acyltransferase porcupine. Nat Chem Biol. 2014; 10:61-8.

24. Takada R, Satomi Y, Kurata T, Ueno N, Norioka S, Kondoh H, Takao T, Takada S. Monounsaturated fatty acid modification of Wnt protein: its role in Wnt secretion. Dev Cell. 2006; 11:791-801.

25. Wilson S, Fan L, Sahgal N, Qi J, Filipp FV. The histone demethylase KDM3A regulates the transcriptional program of the androgen receptor in prostate cancer cells. Oncotarget. 2017; 8:30328-30343. https://doi.org/10.18632/ oncotarget. 15681

26. Eigl BJ, North S, Winquist E, Finch D, Wood L, Sridhar SS, Powers J, Good J, Sharma M, Squire JA, Bazov J, Jamaspishvili T, Cox ME, et al. A phase II study of the HDAC inhibitor SB939 in patients with castration resistant prostate cancer: NCIC clinical trials group study IND195. Invest New Drugs. 2015; 33:969-76.

27. Wheler JJ, Janku F, Falchook GS, Jackson TL, Fu S, Naing A, Tsimberidou AM, Moulder SL, Hong DS, Yang H, PihaPaul SA, Atkins JT, Garcia-Manero G, et al. Phase I study of anti-VEGF monoclonal antibody bevacizumab and histone deacetylase inhibitor valproic acid in patients with advanced cancers. Cancer Chemother Pharmacol. 2014; 73:495-501.

28. Zhang B, Zhao R, He Y, Fu X, Fu L, Zhu Z, Fu L, Dong JT. MicroRNA 100 sensitizes luminal A breast cancer cells to paclitaxel treatment in part by targeting mTOR. Oncotarget. 2016; 7:5702-14. https://doi.org/10.18632/oncotarget.6790. 